PROCEEDINGS OF THE

AMERICAN MATHEMATICAL SOCIETY

Volume 132, Number 2, Pages 353-363

S 0002-9939(03)07164-8

Article electronically published on August 28, 2003

\title{
A PHILOSOPHY FOR THE MODELLING OF REALISTIC NONLINEAR SYSTEMS
}

\author{
PHIL HOWLETT, ANATOLI TOROKHTI, AND CHARLES PEARCE
}

(Communicated by Jonathan M. Borwein)

\begin{abstract}
A nonlinear dynamical system is modelled as a nonlinear mapping from a set of input signals into a corresponding set of output signals. Each signal is specified by a set of real number parameters, but such sets may be uncountably infinite. For numerical simulation of the system each signal must be represented by a finite parameter set and the mapping must be defined by a finite arithmetical process. Nevertheless the numerical simulation should be a good approximation to the mathematical model. We discuss the representation of realistic dynamical systems and establish a stable approximation theorem for numerical simulation of such systems.
\end{abstract}

\section{INTRODUCTION}

To construct a mathematical model of a realistic dynamical system it is necessary to formalize definitions of such crucial physical properties as causality, finite memory and stationarity. The philosophy of realistic systems has been considered by many authors including Russell [1], Paley and Wiener [2], Foures and Segal [3], Falb and Freedman [4, Willems [5], Gohberg [6] and Sandberg and Xu [7. We propose a generic topological structure to describe realistic nonlinear systems and extend the methods of Torokhti and Howlett 8], [9, [10 to prove stable approximation theorems for numerical simulation of these systems. We define a class of $\mathcal{R}$-operators and prove that an $\mathcal{R}$-continuous operator $F$ can be approximated by an $\mathcal{R}$-continuous operator $S$ constructed from an algebra of elementary functions by a finite arithmetic process. The approximation is stable to small disturbances. Our theorem is a generalization of the Stone-Weierstrass theorem. Theorems of this type were extended to operators on topological vector spaces by Prenter 11 and Bruno [12]. A Stone-Weierstrass theory for approximation of continuous functions by superpositions of a sigmoidal function was given by Cybenko [13]. Daugavet 14] considered nonlinear operator approximation by generalized causal operators. We provide a substantial extension of this work and show that our definition of the $\mathcal{R}$-continuous operator includes the accepted notions of causality [1] - 7], 14] and other fundamental realistic properties as special cases. Several key results on operator approximation [11], 12], 114] also follow from particular applications of our main

Received by the editors September 8, 2000.

2000 Mathematics Subject Classification. Primary 47H99, 47A58; Secondary 37M05.

Key words and phrases. Operator approximation, realistic nonlinear systems.

This research was supported by Australian Research Council Grant \#A49943121. 
theorems. In future work we intend to show that certain specific approximation problems [15], [16] can be formulated and solved for $\mathcal{R}$-operators.

\section{Representation of REAListic DYNAMiCAL SYSTEMS}

We define a class of realistic systems. The fundamental idea is that each input or output is uniquely defined by a corresponding legend of historical information. We pay particular attention to systems in which the output history depends continuously on the input history.

\section{1. $\mathcal{R}$-spaces.}

Definition 2.1. 14 Let $X$ and $A$ be Banach spaces and let $\mathcal{L}(X, A)$ be the set of continuous linear operators from $X$ into $A$. Let $T=(T, \rho)$ be a compact metric space and let $\mathcal{M}=\left\{M_{t}\right\}_{t \in T}$ be a family of operators $M_{t} \in \mathcal{L}(X, A)$ with norm $\left\|M_{t}\right\| \leq 1$ for each $t \in T$ and such that $M_{s}[u] \rightarrow M_{t}[u]$ as $\rho(s, t) \rightarrow 0$ for each $u \in X$. The space $X$ equipped with the family of operators $\mathcal{M}$ is called an $\mathcal{R}$-space and is denoted by $X_{\mathcal{R}}=(X, A, T, \mathcal{M})$.

The family $\mathcal{M}$ provides a mechanism for storing and manipulating information about elements in $X$.

Definition 2.2. For each $x \in X$ the collection of elements $\mathcal{M}[x]=\left\{M_{t}[x] \mid t \in\right.$ $T\} \subseteq A$ is called the legend or the complete history of the element $x$. For each $t \in T$ the element $M_{t}[x] \in A$ represents the current history of $x$.

We assume that each element $x \in X$ is uniquely defined by specifying the legend $\mathcal{M}[x]$ of the element 1

Lemma 2.3. $\mathcal{M}[x]=\{0\} \Leftrightarrow x=0$.

If we define $\mathcal{M}[x]+\mathcal{M}[y]=\mathcal{M}[x+y]$ and $\alpha \mathcal{M}[x]=\mathcal{M}[\alpha x]$ for each $\alpha \in \mathbb{C}$, then the set $\mathcal{X}=\mathcal{M}[X]=\{\mathcal{M}[x] \mid x \in X\}$ of all legends is a linear space over $\mathbb{C}$ with zero element $\mathcal{M}[0]$. If we further define $\|\mathcal{M}[x]\|=\sup _{t \in T}\left\|M_{t}[x]\right\|$, then $\mathcal{X}$ is a normed linear space.

Definition 2.4. The archival function $\mathcal{H}: \mathcal{X} \mapsto X$ is a well-defined linear function given by the formula $\mathcal{H}(\mathcal{M}[x])=x$ for all $\mathcal{M}[x] \in \mathcal{X}$.

Definition 2.5. The family $\mathcal{M}$ is said to be pointwise normally extreme on $X$ if, for each $x \in X$, there exists $t=t_{x} \in T$ such that $\left\|M_{t}[x]\right\|=\|x\|$.

Lemma 2.6. If the family $\mathcal{M}$ is pointwise normally extreme on $X$, then the normed linear spaces $\mathcal{X}$ and $X$ are isometrically isomorphic under the archival mapping $\mathcal{H}: \mathcal{X} \mapsto X$.

Corollary 2.7. If the family $\mathcal{M}$ is pointwise normally extreme on $X$, then $\mathcal{X}$ is a Banach space and $\mathcal{H} \in \mathcal{L}(\mathcal{X}, X)$ with $\|\mathcal{H}\|=1$.

\footnotetext{
${ }^{1}$ This is an adaption of the idea that a function is defined by specifying the complete set of function values.
} 


\section{2. $\mathcal{R}$-operators and $\mathcal{R}$-continuous operators.}

Definition 2.8. [14] Let $X_{\mathcal{R}}=(X, A, T, \mathcal{M})$ and $Y_{\mathcal{R}}=(Y, B, T, \mathcal{N})$ be $\mathcal{R}$-spaces, and let the closed set $E \subseteq T \times T$ be an equivalence relation. Let $K \subseteq X$ be a compact set, and let $x \in \bar{K}$ and $t \in T$. The operator $F: K \rightarrow Y$ is an $\mathcal{R}$-operator at $M_{t}[x] \in A$ if $M_{s}[v]=M_{t}[x] \Rightarrow N_{s}[F(v)]=N_{t}[F(x)]$ whenever $(s, t) \in E$ and $v \in K$. If $F: K \mapsto Y$ is an $\mathcal{R}$-operator at $M_{t}[x] \in A$ for all $x \in K$ and $t \in T$, then we say that $F: K \mapsto Y$ is an $\mathcal{R}$-operator.

A dynamical system defined by an $\mathcal{R}$-operator $F: K \mapsto Y$ has the following interpretation. For each $x \in K$ and $t \in T$ the current history $N_{t}[F(x)]$ of the output depends only on the current history $M_{t}[x]$ of the input. For a theory of constructive approximation we require the dependence to be continuous.

Definition 2.9. Let $X_{\mathcal{R}}=(X, A, T, \mathcal{M})$ and $Y_{\mathcal{R}}=(Y, B, T, \mathcal{N})$ be $\mathcal{R}$-spaces, and let the closed set $E \subseteq T \times T$ be an equivalence relation. Let $K \subseteq X$ be compact, and let $x \in K$ and $t \in T$. The operator $F: K \rightarrow Y$ is $\mathcal{R}$-continuous at $M_{t}[x] \in A$ if, for each open neighbourhood of zero $H \subseteq B$, there is an open neighbourhood of zero $G=G(x, t, H) \subseteq A$ such that $M_{s}[v] \in M_{t}[x]+G \Rightarrow N_{s}[F(v)] \in N_{t}[F(x)]+H$ when $(s, t) \in E$ and $v \in K$. If $F: K \mapsto Y$ is $\mathcal{R}$-continuous at $M_{t}[x] \in A$ for all $x \in K$ and $t \in T$, then we say that $F$ is $\mathcal{R}$-continuous.

Lemma 2.10. If $F: K \mapsto Y$ is $\mathcal{R}$-continuous, then $F$ is also an $\mathcal{R}$-operator.

Lemma 2.11. For each $t \in T$ the set $M_{t}[K]=\left\{M_{t}[x] \mid x \in K\right\} \subseteq A$ is compact.

Proof. If $\left\{G_{\gamma}\right\}_{\gamma \in \Gamma}$ is a collection of open sets, then $M_{t}[K] \subseteq \bigcup_{\gamma \in \Gamma} G_{\gamma} \Rightarrow K \subseteq$ $\bigcup_{\gamma \in \Gamma} U_{\gamma}$ where each $U_{\gamma}=M_{t}^{-1}\left[G_{\gamma}\right]$ is also open. Since $K$ is compact there is a finite subcollection $U_{\gamma_{1}}, \ldots, U_{\gamma_{r}}$ such that $K \subseteq \bigcup_{i=1}^{r} U_{\gamma_{i}} \Rightarrow M_{t}[K] \subseteq \bigcup_{i=1}^{r} G_{\gamma_{i}}$.

For each $t \in T$ let $E_{t}=\{s \mid(s, t) \in E\} \subseteq T$. Note that $E_{t}$ is compact. We wish to show that the set $\mathcal{M}_{t}[K]=\left\{M_{s}[K] \mid s \in E_{t}\right\}$ is also compact.

Lemma 2.12. Let $s \in T$. If $M_{s}[K] \subseteq G$ where $G$ is an open set, then we can find $\delta=\delta(s, G)>0$ such that $M_{r}[K] \subseteq G$ when $\rho(r, s)<\delta$.

Proof. If not $\exists$ sequences $\left\{r_{i}\right\} \subseteq T$ with $\rho\left(r_{i}, s\right) \rightarrow 0$ and $\left\{u_{i}\right\} \subseteq K$ such that $M_{r_{i}}\left[u_{i}\right] \notin G$ for each $i$. We can assume $u_{i} \rightarrow v$ for some $v \in K$. Choose $\alpha>0$ and $G_{\alpha}=\{a \mid\|a\|<\alpha\} \subseteq A$ so that $M_{s}[v]+G_{\alpha} \subseteq G$. If $U_{\alpha}=\{u \mid\|u\|<\alpha\} \subseteq X$, then $u \in U_{\alpha} / 2 \Rightarrow M_{r}[u] \in G_{\alpha} / 2$. If $i$ is so large that $u_{i}-v \in U_{\alpha} / 2$ and $M_{r_{i}}[v] \in$ $M_{s}[v]+G_{\alpha} / 2$, then $M_{r_{i}}\left[u_{i}\right] \in M_{s}[v]+G_{\alpha} \subseteq G$. This is a contradiction.

Lemma 2.13. For each $t \in T$ the set $\mathcal{M}_{t}[K]$ is a compact subset of $A$.

Proof. Let $t \in T$ and $s \in E_{t}$ and suppose that $\left\{G_{\gamma}\right\}_{\gamma \in \Gamma}$ is a collection of open sets with $\mathcal{M}_{t}[K] \subseteq \bigcup_{\gamma \in \Gamma} G_{\gamma}$. Since $M_{s}[K]$ is compact and $M_{s}[K] \subseteq \mathcal{M}_{t}[K]$ for each $s \in E_{t}$ there is a finite subset $\Gamma(s) \subseteq \Gamma$ with $M_{s}[K] \subseteq \bigcup_{\gamma \in \Gamma(s)} G_{\gamma}=G(s)$. Choose $\delta(s)>0$ such that $M_{r}[K] \subseteq G(s)$ whenever $\rho(r, s)<\delta(s)$, and define the open sets $R(s)=\{r \mid \rho(r, s)<\delta(s)\} \subseteq T$ for each $s \in T$. Since $E_{t}$ is compact we know that $E_{t} \subseteq \bigcup_{s \in E_{t}} R(s) \Rightarrow E_{t} \subseteq \bigcup_{j=1}^{q} R\left(s_{j}\right)$ for some finite subcollection $\left\{R\left(s_{j}\right)\right\}_{j=1,2, \ldots, q}$ and since $\bigcup_{r \in R\left(s_{j}\right)} M_{r}[K] \subseteq G\left(s_{j}\right)$ for each $j=1, \ldots, q$ we have

$$
\mathcal{M}_{t}[K]=\bigcup_{r \in E_{t}} M_{r}[K]=\bigcup_{j=1}^{q}\left[\bigcup_{r \in R\left(s_{j}\right)} M_{r}[K]\right] \subseteq \bigcup_{j=1}^{q} G\left(s_{j}\right)=\bigcup_{j=1}^{q}\left[\bigcup_{\gamma \in \Gamma\left(s_{j}\right)} G_{\gamma}\right] \text {. }
$$


Therefore $\mathcal{M}_{t}[K]$ is compact.

Lemma 2.14. Let $F: K \mapsto Y$ be continuous and $\mathcal{R}$-continuous. For each neighbourhood of zero, $H \subseteq B$ there exists a neighbourhood of zero $G=G(H)$ such that $M_{r}[u]-M_{s}[v] \in G \Rightarrow N_{r}[F(u)]-N_{s}[F(v)] \in H$ whenever $(r, s) \in E$ and $u, v \in K$. Hence $F$ is uniformly $\mathcal{R}$-continuous.

Proof. If not then for some $\beta>0$ there exist neighbourhoods of zero $H_{\beta}=\{b \mid$ $\|b\|<\beta\} \subseteq B$ and $G_{1 / n}=\{a \mid\|a\|<1 / n\} \subseteq A$ for each $n=1,2, \ldots$ and $u_{n}, v_{n} \in K$ and $r(n), s(n), t(n) \in T$ with $r(n), s(n) \in E_{t(n)}$ for each $n=1,2, \ldots$ such that $M_{r(n)}\left[u_{n}\right]-M_{s(n)}\left[v_{n}\right] \in G_{1 / n} \quad$ and $\quad N_{r(n)}\left[F\left(u_{n}\right)\right]-N_{s(n)}\left[F\left(v_{n}\right)\right] \notin H_{\beta}$. We suppose, without loss of generality, that there exist $u, v \in K$ with $u_{n} \rightarrow u$ and $v_{n} \rightarrow v$ as $n \rightarrow \infty$ and points $r, s, t \in T$ with $\rho(r(n), r) \rightarrow 0, \rho(s(n), s) \rightarrow 0$ and $\rho(t(n), t) \rightarrow 0$ as $n \rightarrow \infty$. Since $(r(n), t(n)) \in E$ and $(s(n), t(n)) \in E$ and since $E$ is closed, it follows that $(r, t) \in E$ and $(s, t) \in E$. Hence $r, s \in E_{t}$. Choose $\alpha>0$ and define $G_{\alpha}=\{a \mid\|a\|<\alpha\} \subseteq A$. We have $M_{r}[x] \in G_{\alpha} / 5$, whenever $x \in U_{\alpha} / 5$ where $U_{\alpha}=\{x \mid\|x\|<\alpha\} \subseteq X$. If we take $n$ so large that $u-u_{n}, v-v_{n} \in U_{\alpha} / 5, \quad M_{r(n)}[u]-M_{r}[u], M_{s(n)}[v]-M_{s}[v] \in G_{\alpha} / 5$ and $\quad G_{1 / n} \subseteq$ $G_{\alpha} / 5$ then $M_{r}[u]-M_{s}[v] \in G_{\alpha}$. Since $\alpha$ is arbitrary it follows that $M_{r}[u]-M_{s}[v]=0$ and since $r, s \in E_{t}$ the $\mathcal{R}$-continuity of $F$ implies that $N_{r}[F(u)]-N_{s}[F(v)]=0$. Define $V_{\beta}=\{y \mid\|y\|<\beta\} \subseteq Y$. Note that $N_{r}[y] \in H_{\beta} / 4$ whenever $y \in V_{\beta} / 4$. Choose $n$ so large that $F\left(u_{n}\right)-F(u), F\left(v_{n}\right)-F(v) \in V_{\beta} / 4$ and $\quad N_{r(n)}[F(u)]-$ $N_{r}[F(u)], N_{s(n)}[F(v)]-N_{s}[F(v)] \in H_{\beta} / 4$. Hence $N_{r(n)}\left[F\left(u_{n}\right)\right]-N_{s(n)}\left[F\left(v_{n}\right)\right] \in H_{\beta}$, which is a contradiction.

2.3. The collection of auxiliary mappings. To establish a constructive approximation for $\mathcal{R}$-continuous mappings we define a collection of auxiliary mappings.

Definition 2.15. Let $F: K \mapsto Y$ be $\mathcal{R}$-continuous. For each $t \in T$ define the auxiliary mapping $f_{t}: \mathcal{M}_{t}[K] \mapsto B$ by setting $f_{t}\left(M_{s}[v]\right)=N_{s}[F(v)]$ for each $s \in E_{t}$ and $v \in K$.

This is a good definition because $M_{r}[u]=M_{s}[v] \Rightarrow N_{r}[F(u)]=N_{s}[F(v)]$ for each $r, s \in E_{t}$ and each $u, v \in K$. The mapping $f_{t}: \mathcal{M}_{t}[K] \mapsto B$ is continuous at each point $M_{s}[v] \in \mathcal{M}_{t}[K]$ because, for each open neighbourhood of zero $H \subseteq B$, there is a corresponding open neighbourhood of zero $G=G_{t}(v, s, H)=G(v, s, H) \cap$ $\mathcal{M}_{t}[K] \subseteq A$ such that $M_{r}[u]-M_{s}[v] \in G \Rightarrow f_{t}\left(M_{r}[u]\right)-f_{t}\left(M_{s}[v]\right)=N_{r}[F(u)]-$ $N_{s}[F(v)] \in H$ whenever $r \in E_{t}$ and $u \in K$. Because $\mathcal{M}_{t}[K]$ is compact the mapping $f_{t}: \mathcal{M}_{t}[K] \mapsto B$ is uniformly continuous and for each neighbourhood of zero $H \subseteq B$, there is a neighbourhood of zero $G=G_{t}(H) \subseteq A$ such that $M_{r}[u]-M_{s}[v] \in G \Rightarrow f_{t}\left(M_{r}[u]\right)-f_{t}\left(M_{s}[v]\right) \in H$ whenever $r, s \in E_{t}$ and $u, v \in K$. Lemma 2.14 shows that when $F: K \mapsto Y$ is continuous the collection $\left\{f_{t}\right\}_{t \in T}$ is uniformly equi-continuous. That is, for each neighbourhood of zero $H \subseteq B$ there is a neighbourhood of zero $G=G(H) \subseteq A$ such that for all $t \in T$ we have $M_{r}[u]-M_{s}[v] \in G \Rightarrow f_{t}\left(M_{r}[u]\right)-f_{t}\left(M_{s}[v]\right) \in H$ whenever $r, s \in E_{t}$ and $u, v \in K$.

2.4. Some examples of $\mathcal{R}$-continuous operators. The following theorem of $\mathrm{M}$. Riesz is used in the examples to justify compactness of the set $K$.

Theorem 2.16. Let $K \subseteq L^{p}([0,1])$ and write $\mathcal{T}_{h} x(r)=x(r+h) \forall x \in K ; r, r+h \in$ $[0,1]$. The set $K$ is compact if and only if $\exists M>0$ with $\|x\|_{p} \leq M$ and $\delta=\delta(\epsilon)$ such that $\left\|\mathcal{T}_{h} x-x\right\|_{p}<\epsilon$ whenever $|h|<\delta$ for all $x \in K$. 
2.4.1. A causal operator. Let $X=L^{1}([0,1]), K=\{x|| x(s)-x(t)|\leq| s-t \mid \forall s, t \in$ $[0,1]\} \subseteq X$ and $Y=C([0,1])$. The operator $F: K \mapsto Y$ is a $\mathcal{C}$-operator on the time interval $T=[0,1]$ if, for all $t \in T$ and $u, x \in K,\{u(s)=x(s) \forall s \in[0, t]\} \Rightarrow$ $\{[F(u)](s)=[F(x)](s) \forall s \in[0, t]\}$. Note that the output at time $t$ depends only on the input prior to time $t$. The operator $F$ is uniformly $\mathcal{C}$-continuous if, for all $t \in T$ and all $u, x \in K$ and for each $\beta>0$, we can find $\alpha=\alpha(\beta)>0$ such that $\left\{\left|\int_{[0, s]} u(r) d r-\int_{[0, s]} x(r) d r\right|<\alpha \forall s \in[0, t]\right\} \Rightarrow\{|[F(u)](s)-[F(x)](s)|<$ $\beta \forall s \in[0, t]\}$. To show that a uniformly $\mathcal{C}$-continuous operator is a special case of a uniformly $\mathcal{R}$-continuous operator, set $A=B=C(T)$ and $\tau=\min (s, t)$ and define $M_{t}[x](s)=\int_{[0, \tau]} x(r) d r \quad$ and $\quad N_{t}[y](s)=y(\tau)$ for $x \in X, y \in Y$ and $s, t \in T$. Let $E=\{(t, t) \mid t \in T\}$. In this notation $F$ is a uniformly $\mathcal{C}$-continuous operator on $T$ if and only if for all $t \in T$, and all $u, x \in K$ and for each $\beta>0$, we can find $\alpha=\alpha(\beta)$ such that $\left\{\left\|M_{t}[u]-M_{t}[x]\right\|<\alpha\right\} \Rightarrow\{\|[F(u)]-[F(x)]\|<\beta\}$, which, in turn, is equivalent to saying that $F$ is a uniformly $\mathcal{R}$-continuous operator on $T$. For a particular instance we note that the operator $F_{C}: K \mapsto Y$ defined by $\left[F_{C}(x)\right](t)=e^{-t} \int_{[0, t]} e^{s} x(s) d s$ for each $t \in T$ is a uniformly $\mathcal{C}$-continuous operator.

2.4.2. A stationary operator with finite memory. Let $X=L^{\infty}(\mathbb{R}), K=\{x \mid x(t)=$ 0 for $t \notin[0,1]$ and $|x(s)-x(t)| \leq|s-t| \forall s, t \in \mathbb{R}\} \subseteq X$ and $Y=C(\mathbb{R})$. The operator $F: K \mapsto Y$ is a stationary operator with finite memory $\Delta>0$ on the time interval $T=[0,1+\Delta]$ if, for all $u, x \in K$ and all $s, t \in T,\{u(s+r-\Delta)=x(t+r-\Delta) \forall r \in$ $[0, \Delta]\} \Rightarrow\{[F(u)](s)=[F(x)](t)\}$. The output at time $t$ depends only on the inputs at times $s \in[t-\Delta, t]$. We say that $F$ is an $\mathcal{S}$-operator. The operator $F$ is uniformly $\mathcal{S}$-continuous on $T$ if, $\forall\{u, x \in K ; s, t \in T ; \beta>0\}$, we can find $\alpha=\alpha(\beta)>0$ such that $\{|u(s+r-\Delta)-x(t+r-\Delta)|<\alpha \forall r \in[0, \Delta]\} \Rightarrow\{|[F(u)](s)-[F(x)](t)|<\beta\}$. To show that a uniformly $\mathcal{S}$-continuous operator is a special case of a uniformly $\mathcal{R}$-continuous operator, set $A=L^{\infty}([0, \Delta])$ and $B=C([0,1+\Delta])$. For each $t \in T$ define $M_{t}: X \mapsto A$ by $M_{t}[x](r)=x(r+t-\Delta) \forall r \in[0, \Delta]$ and $N_{t}: Y \mapsto$ $C([0,1+\Delta])$ by $N_{t}[y](r)=y(t) \forall r \in[0,1+\Delta]$. Let $E=T \times T$. The operator $F$ is uniformly $\mathcal{S}$-continuous on $T$ if and only if for all $u, x \in K$ and all $s, t \in T$ and for each $\beta>0$, we can find $\alpha=\alpha(\beta)>0$ such that $\left\{\left\|M_{s}[u]-M_{t}[x]\right\|<\alpha\right\} \Rightarrow$ $\left\{\left\|N_{s}[F(u)]-N_{t}[F(x)]\right\|<\beta\right\}$, which is equivalent to saying that $F$ is a uniformly $\mathcal{R}$-continuous operator on $T$. In particular, the mapping $F_{\Delta}: K \mapsto Y$ defined by $\left[F_{\Delta}(x)\right](t)=\frac{1}{\Delta} \int_{[t-\Delta, t]} x(r) d r$ for each $x \in X$ and $t \in \mathbb{R}$ is a uniformly $\mathcal{S}$-continuous operator.

\section{The MOdulus of CONTINUITY}

Definition 3.1. Let $X$ and $Y$ be separable Banach spaces. Let $K \subseteq X$ be a compact set, and let $F: K \rightarrow Y$ be a continuous map. The modulus of continuity $\omega=\omega[F]: \mathbb{R}_{+} \rightarrow \mathbb{R}_{+}$is given by the formula

$$
\omega(\delta)=\sup _{x_{1}, x_{2} \in K,\left\|x_{1}-x_{2}\right\| \leq \delta}\left\|F\left(x_{1}\right)-F\left(x_{2}\right)\right\| .
$$

Note that $\omega(0)=0$ and $\omega(\delta) \leq \omega\left(\delta^{\prime}\right)$ whenever $\delta \leq \delta^{\prime}$. We will show that $\omega$ is a uniformly continuous function.

Lemma 3.2. Let $X$ and $Y$ be separable Banach spaces. Let $K \subseteq X$ be a compact set and $F: K \rightarrow Y$ a continuous map. Let $\omega=\omega[F]: \mathbb{R}_{+} \rightarrow \mathbb{R}_{+}$be the corresponding 
modulus of continuity. Then for each $\tau>0$ we can find $\sigma=\sigma(\tau)>0$ such that $0 \leq \omega\left(\delta^{\prime}\right)-\omega(\delta) \leq \tau$ whenever $0 \leq \delta^{\prime}-\delta \leq \sigma$.

Proof. Define $\Delta F: K \times K \mapsto Y$ by setting $\Delta F(x)=F\left(x_{2}\right)-F\left(x_{1}\right)$ for each $x=\left(x_{1}, x_{2}\right) \in K$. Clearly $\Delta F$ is continuous with respect to the norm $\|x\|_{K \times K}=$ $\left\|x_{1}\right\|+\left\|x_{2}\right\|$ and hence, since $K \times K$ is compact, $\Delta F$ is uniformly continuous. If we define $D_{\delta}=\left\{x \mid\left\|x_{2}-x_{1}\right\| \leq \delta\right\}$, then $D_{\delta} \subseteq K \times K$ is compact and $\omega(\delta)=\sup _{x \in D_{\delta}}\|\Delta F(x)\|$ for each $\delta \geq 0$. Fix $\tau>0$ and choose $\sigma=\sigma(\tau)>0$ such that $\left\|\Delta F\left(x^{\prime}\right)-\Delta F(x)\right\|<\tau$ whenever $\left\|x^{\prime}-x\right\|_{K \times K}<\sigma$. Now suppose that $0 \leq \delta^{\prime}-\delta \leq \sigma$. Find $x^{\prime} \in D_{\delta^{\prime}}$ with $x_{2}^{\prime} \neq x_{1}^{\prime}$ and $\omega\left(\delta^{\prime}\right)=\left\|\Delta F\left(x^{\prime}\right)\right\|$, and define $\theta \in$ $[0,1]$ so that $\theta\left\|x_{2}^{\prime}-x_{1}^{\prime}\right\|=\delta$. Let $x^{\prime \prime}=\left(x_{2}^{\prime}, x_{1}^{\prime}\right)$ and define $x=\theta x^{\prime}+(1-\theta)\left(x^{\prime}+x^{\prime \prime}\right) / 2$. It is easy to see that $\left\|x_{2}-x_{1}\right\|=\delta$ and that $\left\|x^{\prime}-x\right\|_{K \times K} \leq \sigma$. It follows that $\omega\left(\delta^{\prime}\right)=\left\|\Delta F\left(x^{\prime}\right)\right\| \leq\|\Delta F(x)\|+\tau \leq \omega(\delta)+\tau$. Thus $0 \leq \omega\left(\delta^{\prime}\right)-\omega(\delta) \leq \tau$ when $0 \leq \delta^{\prime}-\delta \leq \sigma$ and hence $\omega$ is uniformly continuous on $\mathbb{R}_{+}$.

3.1. The $\mathcal{R}$-modulus of continuity. The $\mathcal{R}$-modulus of continuity will be used to characterize our constructive approximation theorems for $\mathcal{R}$-continuous operators.

Definition 3.3. 14 Let $X_{\mathcal{R}}=\{X, A, T, \mathcal{M}\}$ and $Y_{\mathcal{R}}=\{Y, B, T, \mathcal{N}\}$ be $\mathcal{R}$-spaces, and let $E \subseteq T \times T$ be the given equivalence relation. Let $K \subseteq X$ be a compact set, and suppose that the map $F: K \rightarrow Y$ is $\mathcal{R}$-continuous. The function $\omega_{\mathcal{R}}=$ $\omega_{\mathcal{R}}[F]: \mathbb{R}_{+} \rightarrow \mathbb{R}_{+}$defined by

$$
\omega_{\mathcal{R}}(\delta)=\sup _{\substack{u, v \in K ;(r, s) \in E: \\\left\|M_{r}[u]-M_{s}[v]\right\| \leq \delta}}\left\|N_{r}[F(u)]-N_{s}[F(v)]\right\|
$$

is called the $\mathcal{R}$-modulus of continuity of the operator $F$.

Definition 3.4. We say that $\left(X_{\mathcal{R}}, Y_{\mathcal{R}}\right)$ is a complete $\mathcal{R}$-pair if $E=T \times T$ and an incomplete $\mathcal{R}$-pair if $E \neq T \times T$.

Lemma 3.5. Let $\left(X_{\mathcal{R}}, Y_{\mathcal{R}}\right)$ be a complete $\mathcal{R}$-pair and suppose that $F: K \mapsto Y$ is $\mathcal{R}$-continuous. Then the $\mathcal{R}$-modulus of continuity $\omega_{\mathcal{R}}=\omega_{\mathcal{R}}[F]: \mathbb{R}_{+} \rightarrow \mathbb{R}_{+}$is uniformly continuous with $\omega_{\mathcal{R}}(0)=0$.

Proof. Since $E_{t}=T$ for all $t \in T$ it follows that $\mathcal{M}[K]=\mathcal{M}_{t}[K]=\left\{M_{s}[x] \mid x \in\right.$ $K$ and $s \in T\} \subseteq A$ for all $t \in T$. Define an auxiliary mapping $f: \mathcal{M}[K] \mapsto B$ by setting $f\left(M_{t}[x]\right)=N_{t}[F x]$ for each $x \in K$ and $t \in T$. Recall from our earlier remarks that the mapping $f: \mathcal{M}[K] \mapsto B$ is uniformly continuous. The function $\omega_{f}: \mathbb{R}_{+} \mapsto \mathbb{R}_{+}$is the modulus of continuity of $f$. Lemma 3.2 shows that $\omega_{f}$ is uniformly continuous. Since $\omega_{f}(\delta)=\omega_{\mathcal{R}}(\delta)$ we obtain the desired result.

Lemma 3.6. Let $\left(X_{\mathcal{R}}, Y_{\mathcal{R}}\right)$ be an incomplete $\mathcal{R}$-pair and suppose that $F: K \mapsto Y$ is both continuous and $\mathcal{R}$-continuous. Then the $\mathcal{R}$-modulus of continuity $\omega_{\mathcal{R}}=$ $\omega_{\mathcal{R}}[F]: \mathbb{R}_{+} \rightarrow \mathbb{R}_{+}$is uniformly continuous with $\omega_{\mathcal{R}}(0)=0$.

Proof. Since $\left(X_{\mathcal{R}}, Y_{\mathcal{R}}\right)$ is an incomplete $\mathcal{R}$-pair we consider the various equivalence classes $E_{t}$ for each $t \in T$. We saw earlier that for each $t \in T$ there is an auxiliary mapping $f_{t}: M_{t}[K] \mapsto B$ defined by $f_{t}\left(M_{t}[x]\right)=N_{t}[F(x)]$ for all $x \in K$. Let $\omega\left[f_{t}\right]$ : $\mathbb{R}_{+} \mapsto \mathbb{R}_{+}$be the modulus of continuity for the map $f_{t}$, and consider the argument used in Lemma 3.2. Define $\Delta f_{t}: M_{t}[K] \times M_{t}[K]$ by the formula $\Delta f_{t}(p, q)=\| f_{t}(p)-$ $f_{t}(q) \|$ for each $(p, q) \in M_{t}[K] \times M_{t}[K]$. Choose $\tau>0$. From our earlier remarks about the uniform equi-continuity of the family of auxiliary mappings $\left\{f_{t}\right\}_{t \in T}$, we can choose $\sigma=\sigma(\tau)>0$ such that for all $t \in T$ we have $\left\|\Delta f_{t}\left(p^{\prime}, q^{\prime}\right)-\Delta f_{t}(p, q)\right\|<\tau$ 
whenever $\left\|\left(p^{\prime}, q^{\prime}\right)-(p, q)\right\|<\sigma$. Now it is clear from Lemma 3.2 that for all $t \in T$ we have $0 \leq \omega\left[f_{t}\right]\left(\delta^{\prime}\right)-\omega\left[f_{t}\right](\delta) \leq \tau$ whenever $0 \leq \delta^{\prime}-\delta \leq \sigma$. Thus the family $\left\{\omega\left[f_{t}\right]\right\}_{t \in T}$ is also uniformly equi-continuous. Since $\omega_{\mathcal{R}}(\delta)=\sup _{t \in T} \omega\left[f_{t}\right](\delta)$, it follows that $0 \leq \omega_{\mathcal{R}}\left(\delta^{\prime}\right)-\omega_{\mathcal{R}}(\delta) \leq \tau$ whenever $0 \leq \delta^{\prime}-\delta \leq \sigma$.

\section{Approximation of NONLINEAR OPERATORS ON COMPACT SETS}

We describe briefly the recent work by Torokhti and Howlett [8]. Let $X, Y$ be locally convex topological vector spaces and let $K \subseteq X$ be a compact subset. Let $F: K \subseteq X \rightarrow Y$ be a continuous map. If $F$ is known only on $K$, then for some suitable neighbourhood $\epsilon$ of zero in $X$ the construction of an extended operator $S: K+\epsilon \subseteq X \rightarrow Y$ is an important ingredient in the approximation procedure. The extension of the domain allows consideration of a small disturbance in the input signal. Such disturbances are unavoidable in the modelling process. The main result is formulated as follows. Let $X, Y$ be topological vector spaces with the Grothendieck property of approximation 3 and with approximating sequences $\left\{G_{m}\right\}_{m=1,2, \ldots} \subseteq \mathcal{L}\left(X, X_{m}\right),\left\{H_{n}\right\}_{n=1,2, \ldots} \subseteq \mathcal{L}\left(Y, Y_{n}\right)$ of continuous linear operators, where $X_{m} \subseteq X, Y_{n} \subseteq Y$ are subspaces of dimension $m, n$. Write $X_{m}=\left\{x_{m} \in X \mid\right.$ $\left.x_{m}=\sum_{j=1}^{m} a_{j} u_{j}\right\}$ and $Y_{n}=\left\{y_{n} \in Y \mid y_{n}=\sum_{k=1}^{n} b_{k} v_{k}\right\}$, where $a \in \mathbb{R}^{m}, b \in \mathbb{R}^{n}$ and $\left\{u_{j}\right\}_{j=1,2, \ldots, m},\left\{v_{k}\right\}_{k=1,2, \ldots, n}$ are bases in $X_{m}, Y_{n}$ respectively. Let $\mathcal{G}=\{g\}$ be an algebra of continuous functions $g: \mathbb{R}^{m} \rightarrow \mathbb{R}$ that satisfies the conditions of Stone's algebra. Define the operators $Q \in \mathcal{L}\left(X_{m}, \mathbb{R}^{m}\right), Z: \mathbb{R}^{m} \rightarrow \mathbb{R}^{n}$ and $W \in \mathcal{L}\left(\mathbb{R}^{n}, Y_{n}\right)$ by $Q\left(x_{m}\right)=a, \quad Z(a)=\left(g_{1}(a), g_{2}(a), \ldots, g_{n}(a)\right)$ and $W(z)=\sum_{k=1}^{n} z_{k} v_{k}$ where each $g_{k} \in \mathcal{G}$ and $z_{k}=g_{k}(a)$. Subject to an appropriate choice of the functions $\left\{g_{k}\right\} \in \mathcal{G}$, so that $z_{k}$ provides a sufficiently good approximation to $b_{k}$, the following stable approximation theorems can be established.

Theorem 4.1. Let $X, Y$ be locally convex topological vector spaces as above, and let $X$ be normal. Let $K \subseteq X$ be a compact set and $F: K \rightarrow Y$ a continuous map. For a given convex neighbourhood of zero $\tau \subseteq Y$ there exists a neighbourhood of zero $\sigma \subseteq X$, an associated continuous operator $S: X \rightarrow Y_{n}$ defined by finite arithmetic in the form $S=S_{\sigma}=W Z Q G_{m}$ and a neighbourhood of zero $\epsilon \subseteq X$ such that for all $x \in K$ and all $x^{\prime} \in X$ with $x^{\prime}-x \in \epsilon$ we have $F(x)-S\left(x^{\prime}\right) \in \tau$.

Theorem 4.2. Let $X$ and $Y$ be separable Banach spaces. Let $K \subseteq X$ be a compact set and $F: K \rightarrow Y$ a continuous map. For any given numbers $\delta>0$ and $\tau>0$ and for all $x \in K$ and all $x^{\prime} \in X$ with $\left\|x^{\prime}-x\right\| \leq \delta$, there exists an operator $S=W Z Q G_{m}: X \mapsto Y$ defined by finite arithmetic such that $\left\|F(x)-S\left(x^{\prime}\right)\right\| \leq$ $\frac{1}{2} \omega[F](2 \delta)+\tau$.

Proof. The proof of the latter result uses an argument proposed by Daugavet 14 . Since 14 is difficult to obtain, the proof is given in Appendix A.

4.1. A model for constructive approximation in the class of $\mathcal{R}$-continuous operators. When $F$ is an $\mathcal{R}$-continuous operator we prove the existence of an approximating $\mathcal{R}$-continuous operator $S$ that is stable to small disturbances. The operator $S$ defines a model of the real system and is constructed from an algebra of elementary continuous functions by a process of finite arithmetic.

\footnotetext{
${ }^{2}$ The space $X$ possesses the Grothendieck property of approximation if there is a sequence $\left\{G_{m}\right\}_{m \in \mathbb{N}} \subseteq \mathcal{L}\left(X, X_{m}\right)$ where $X_{m} \subseteq X$ is a subspace of dimension $m$ and the operators $G_{m}$ are equi-continuous on compacta and uniformly convergent to unit operators on those compacta.
} 
Theorem 4.3. Let $A$ and $B$ be Banach spaces with the Grothendieck property of approximation, and let $X_{\mathcal{R}}=(X, A, T, \mathcal{M})$ and $Y_{\mathcal{R}}=(Y, B, T, \mathcal{N})$ be $\mathcal{R}$-spaces. Suppose that $\left(X_{\mathcal{R}}, Y_{\mathcal{R}}\right)$ is a complete $\mathcal{R}$-pair and that $\mathcal{N}$ is pointwise normally extreme on $Y$. Let $K \subseteq X$ be a compact set, and let the map $F: K \mapsto Y$ be an $\mathcal{R}$-continuous operator. Then for any fixed real numbers $\delta>0$ and $\tau>0$ there exists an associated $\mathcal{R}$-continuous operator $S$ defined by finite arithmetic in the form $S=W Z Q G: X \mapsto Y$ such that for all $x \in K$ and $x^{\prime} \in X$ with $\left\|x-x^{\prime}\right\| \leq \delta$ we have $\left\|F(x)-S\left(x^{\prime}\right)\right\| \leq \frac{1}{2} \omega_{\mathcal{R}}(2 \delta)+\tau$.

Proof. We recall from Lemma 3.5 that the auxiliary mapping $f: \mathcal{M}[K] \mapsto B$ is uniformly continuous. We will construct a mapping $\sigma: A \rightarrow B$ in the form $\sigma=\pi \nu \lambda \theta$ where $A_{m} \subseteq A$ is a subspace of dimension $m$ and $B_{n} \subseteq B$ is a subspace of dimension $n$, and where $\theta \in \mathcal{L}\left(A, A_{m}\right)$ and $\lambda \in \mathcal{L}\left(A_{m}, \mathbb{R}^{m}\right)$, where $\nu: \mathbb{R}^{m} \mapsto \mathbb{R}^{n}$ is continuous and where $\pi \in \mathcal{L}\left(\mathbb{R}^{n}, B_{n}\right)$. By Theorem 4.2 there exists a continuous mapping $\sigma: A \mapsto B$ in the above form such that for all $w \in \mathcal{M}[K]$ and all $w^{\prime}$ with $\left\|w-w^{\prime}\right\|<\delta$ we have $\left\|f(w)-\sigma\left(w^{\prime}\right)\right\| \leq \frac{1}{2} \omega_{\mathcal{R}}(2 \delta)+\tau$, where we have used the fact that the modulus of continuity of $f$ satisfies $\omega_{f}(\alpha)=\omega_{\mathcal{R}}(\alpha)$ for all $\alpha \in \mathbb{R}_{+}$. Now define $S: X \mapsto Y$ by setting $N_{t}[S x]=\sigma\left(M_{t}[x]\right)$ for each $x \in X$ and each $t \in T$. Our indirect definition assumes that if $N_{t}[y] \in B$ is known for all $t \in T$, then $y \in Y$ is also known. We will follow our earlier notation and write $y=\mathcal{K}(\mathcal{N}[y])$ where $\mathcal{K}: \mathcal{Y} \mapsto Y$ is the appropriate archival function. The mapping $\sigma: A \mapsto B$ is continuous and hence $S: X \mapsto Y$ is an $\mathcal{R}$-continuous operator. Since $\left\|M_{t}\left[x-x^{\prime}\right]\right\| \leq\left\|x-x^{\prime}\right\|$, it follows that $\left\|N_{t}\left[F x-S x^{\prime}\right]\right\|=\left\|f\left(M_{t}[x]\right)-\sigma\left(M_{t}\left[x^{\prime}\right]\right)\right\|<\frac{1}{2} \omega_{\mathcal{R}}(2 \delta)+\tau$ for all $t \in T$ whenever $x \in K$ and $\left\|x-x^{\prime}\right\|<\delta$. But we can choose $t=t_{\left[F(x)-S\left(x^{\prime}\right)\right]} \in T$ such that $\left\|N_{t}\left[F x-S x^{\prime}\right]\right\|=\left\|F(x)-S\left(x^{\prime}\right)\right\|$ and so $\left\|F(x)-S\left(x^{\prime}\right)\right\|<\frac{1}{2} \omega_{\mathcal{R}}(2 \delta)+\tau$ whenever $x \in K$ and $\left\|x-x^{\prime}\right\|<\delta$. Since we defined $N_{t}[S x]=\pi \nu \lambda \theta M_{t}[x]$ we can now write $\mathcal{N}[S x]=\pi \nu \lambda \theta \mathcal{M}[x]$ or, equivalently, $S(x)=\mathcal{K} \pi \nu \lambda \theta \mathcal{H}^{-1}(x)$ for each $x \in X$. Note that $\left\|\mathcal{H}^{-1}\right\| \leq 1$ and that $\|\mathcal{K}\|=1$. If we define $G=\theta \mathcal{H}^{-1}, Q=\lambda, Z=\nu$ and $W=\mathcal{K} \pi$, then we can see that $S$ has the desired form. We assume that $G$ and $W$ can be defined by finite arithmetic or replaced by suitable approximations.

Lemma 4.4. Let $K \subseteq X$ be a compact set. Then for each $\epsilon>0$ we can find $\delta>0$ such that $\left\|M_{s}[x]-M_{t}[x]\right\|<\epsilon$ for all $x \in K$ whenever $s, t \in T$ and $\rho(s, t)<\delta$.

Theorem 4.5. Let $A$ and $B$ be Banach spaces with the Grothendieck property of approximation. Let $X_{\mathcal{R}}=(X, A, T, \mathcal{M})$ and $Y_{\mathcal{R}}=(Y, B, T, \mathcal{N})$ be $\mathcal{R}$-spaces and suppose that $\left(X_{\mathcal{R}}, Y_{\mathcal{R}}\right)$ is an incomplete $\mathcal{R}$-pair and that $\mathcal{N}$ is pointwise normally extreme on $Y$. Let $K \subseteq X$ be a compact set and let the map $F: K \mapsto Y$ be continuous and $\mathcal{R}$-continuous. Then for any fixed real numbers $\delta>0$ and $\tau>0$ there exists an associated operator $S: X \mapsto Y$ defined by $N_{t}[S u]=\sum_{j=1}^{N} \psi_{j}(t) N_{t}\left[S_{j} u\right]$ where $\psi_{j}: T \mapsto \mathbb{R}$ for each $j=1,2, \ldots, N$ and $\left\{\psi_{1}, \ldots, \psi_{N}\right\}$ is a partition of unity and where $S_{j}=W_{j} Z_{j} Q_{j} G_{j}: X \mapsto Y$ for each $j=1,2, \ldots, N$ and each $u \in K$ and $t \in T$. The mapping $S$ is continuous and $\mathcal{R}$-continuous and is defined by a process of finite arithmetic in such a way that for all $x \in K$ and $x^{\prime} \in X$ with $\left\|x-x^{\prime}\right\| \leq \delta$ we have $\left\|F(x)-S\left(x^{\prime}\right)\right\| \leq \frac{1}{2} \omega_{\mathcal{R}}(2 \delta)+\tau$.

Proof. Let $t \in T$ and consider the auxiliary mappings $f_{t}: M_{t}[K] \mapsto B$ and the associated moduli of continuity $\omega\left[f_{t}\right]: \mathbb{R}_{+} \mapsto \mathbb{R}_{+}$. We recall from Lemmas 2.14 and [3.6] that the families $\left\{f_{t}\right\}_{t \in T}$ and $\left\{\omega\left[f_{t}\right]\right\}_{t \in T}$ are each uniformly equi-continuous. Hence, for the given $\tau>0$, it is possible to choose $\epsilon=\epsilon(\tau)>0$ so small that 
$\lambda \leq \delta+\epsilon \Rightarrow \omega_{\mathcal{R}}(2 \lambda) \leq \omega_{\mathcal{R}}(2 \delta)+\tau$ and $\left\|M_{r}[u]-M_{s}[v]\right\|<\epsilon \Rightarrow \| N_{r}[F u]-$ $N_{s}[F v] \|<\tau / 12$ whenever $(r, s) \in E$ and $u, v \in K$. Since $K$ and $F(K)$ are both compact, we can use Lemma 4.4 to find $\gamma>0$ so that both $\left\|M_{s}[x]-M_{t}[x]\right\|<\epsilon$ and $\left\|N_{s}[F x]-N_{t}[F x]\right\|<\tau / 4$ for all $x \in K$ when $\rho(s, t)<\gamma$. Choose a $\gamma$-net $\left\{t_{1}, \ldots, t_{N}\right\} \subseteq T$ such that whenever $t \in T$ we can always find some $j=j(t)$ with $\left\|t-t_{j}\right\|<\gamma$ and let $\left\{\psi_{1}(t), \ldots, \psi_{N}(t)\right\}$, where $\psi_{j}: T \mapsto \mathbb{R}$ for each $j=1,2, \ldots, N$, be a partition of unity on $T$ such that $\psi_{1}, \ldots, \psi_{N} \in C(T), \psi_{j}(t) \geq 0$ for all $t \in T$, $\sum_{j=1}^{N} \psi_{j}(t)=1$ for all $t \in T$, and $\psi_{j}(t)=0$ whenever $\rho\left(t, t_{j}\right) \geq \gamma$. Let $x \in K$ and choose $u \in X$ with $\|u-x\| \leq \delta$. If $\rho\left(t, t_{j}\right)<\gamma$, then $\left\|M_{t}[u]-M_{t_{j}}[x]\right\| \leq$ $\left\|M_{t}[u-x]\right\|+\left\|M_{t}[x]-M_{t_{j}}[x]\right\| \leq\|u-x\|+\epsilon=\lambda \leq \delta+\epsilon$. By applying Theorem 4.2 we can define a function $\sigma_{j}: A \rightarrow B$ in the form $\sigma_{j}=\pi_{j} \nu_{j} \lambda_{j} \theta_{j}$ such that for all $w \in M_{t_{j}}[K]$ and $w^{\prime}$ with $\left\|w^{\prime}-w\right\|<\lambda$ we have $\left\|f_{j}(w)-\sigma_{j}\left(w^{\prime}\right)\right\|<\frac{1}{2} \omega\left[f_{j}\right](2 \lambda)+\frac{\tau}{4}$. Define $S_{j}: X \rightarrow Y$ by setting $N_{t}\left[S_{j} u\right]=\sigma_{j}\left(M_{t}[u]\right)$ and $S: X \mapsto Y$ by the formula $N_{t}[S u]=\sum_{j=1}^{N} \psi_{j}(t) \sigma_{j}\left(M_{t}[u]\right)$ for all $u \in X$ and $t \in T$. Now for $x \in K, u \in X$ with $\|x-u\|<\delta$ and all $t \in T$ we have

$$
\left\|N_{t}[F x]-N_{t}[S u]\right\|=\left\|\sum_{\rho\left(t, t_{j}\right)<r} \psi_{j}(t)\left[N_{t}[F x]-\sigma_{j}\left(M_{t}[u]\right)\right]\right\| .
$$

We make two observations. Firstly, for $\rho\left(t, t_{j}\right)<r$ we have

$$
\begin{aligned}
\left\|N_{t}[F x]-\sigma_{j}\left(M_{t}[u]\right)\right\| & \leq\left\|N_{t}[F x]-N_{t_{j}}[F x]\right\|+\left\|N_{t_{j}}[F x]-\sigma_{j}\left(M_{t}[u]\right)\right\| \\
& \leq\left\|f_{j}\left(M_{t_{j}}[x]\right)-\sigma_{j}\left(M_{t} u\right)\right\|+\frac{\tau}{4} .
\end{aligned}
$$

Secondly, since $\left\|M_{t_{j}}[x]-M_{t}[u]\right\| \leq \lambda$, it follows that $\left\|f_{j}\left(M_{t_{j}}[x]\right)-\sigma_{j}\left(M_{t}[u]\right)\right\| \leq$ $\frac{1}{2} \omega_{\mathcal{R}}(2 \lambda)+\frac{\tau}{4}$. The desired result can now be established.

\section{Appendix A. Proof of Theorem 4.2}

It is well known that any separable Banach space is isometric and isomorphic to a subspace of the space $C([0,1])$ of continuous functions on the interval $[0,1]$. Thus, without loss of generality, we assume $X=Y=C([0,1])$. Define $\varphi: K \times[0,1] \rightarrow \mathbb{R}$ by setting $\varphi(x, t)=F[x](t)$ for all $t \in[0,1]$. Fix $\delta>0$ and $t \in[0,1]$. For each $u \in$ $K_{\delta}=\{u \mid\|u-x\| \leq \delta$ for some $x \in K\}$ choose $x^{+}[u]=x_{\delta, t}^{+}[u], x^{-}[u]=x_{\delta, t}^{-}[u] \in K$ so that

$$
\varphi_{\delta}^{+}(u, t)=\varphi\left(x^{+}[u], t\right)=\max _{x \in K,\|x-u\| \leq \delta} \varphi(x, t)
$$

and

$$
\varphi_{\delta}^{-}(u, t)=\varphi\left(x^{-}[u], t\right)=\min _{x \in K,\|x-u\| \leq \delta} \varphi(x, t)
$$

and set $\varphi_{\delta}(u, t)=\frac{1}{2}\left[\varphi_{\delta}^{+}(u, t)+\varphi_{\delta}^{-}(u, t)\right]$. Define $F_{\delta}: K_{\delta} \rightarrow C([0,1])$ by setting $F_{\delta}[u](t)=\varphi_{\delta}(u, t)$ for all $\delta>0$ and each $t \in[0,1]$. If $u \in K_{\delta}$ and $x \in K$ with $\|u-x\| \leq \delta$, then $\left|\varphi(x, t)-\varphi_{\delta}(u, t)\right| \leq \omega(2 \delta) / 2$ for all $t \in[0,1]$, and hence it follows that $\left\|F(x)-F_{\delta}(u)\right\| \leq \frac{1}{2} \omega(2 \delta)$. However, $F_{\delta}$ may not be continuous. Therefore for fixed $t \in[0,1]$ and each pair of positive real numbers $\lambda$ and $\mu$ we define

$$
\varphi_{\lambda, \mu}(u, t)=\frac{1}{2 \mu} \int_{[\lambda, \lambda+\mu]}\left[\varphi_{\xi}^{+}(u, t)+\varphi_{\xi}^{-}(u, t)\right] d \xi
$$


and $F_{\lambda, \mu}: K_{\lambda} \rightarrow C([0,1])$ by setting $F_{\lambda, \mu}[u](t)=\varphi_{\lambda, \mu}(u, t)$ for all $t \in[0,1]$. If $\|u-v\|<\rho$, then it can be shown that

$$
\left\|F_{\lambda, \mu}[u]-F_{\lambda, \mu}[v]\right\| \leq \frac{2 \rho F_{K}}{\mu}
$$

where $F_{K}=\max _{x \in K}\|F(x)\|$. This shows that the operator $F_{\lambda, \mu}$ is continuous. If $x \in K$ and $\|x-u\|<\lambda$, then it follows that $\left\|F(x)-F_{\lambda, \mu}(u)\right\| \leq \frac{1}{2} \omega(2 \nu)$ where $\nu=\lambda+\mu$. To prove the desired result we take $\tau>0$ and choose $\epsilon>0$ so that $\omega(2 \delta+\epsilon) \leq \omega(2 \delta)+\tau$ for all $\delta>0$. Now we set $\lambda=\delta+\epsilon / 2$ and $\mu=\epsilon / 2$ and note that if $\|x-u\| \leq \lambda$, then $\left\|F(x)-F_{\lambda, \mu}(u)\right\| \leq \frac{1}{2} \omega(2 \delta)+\frac{\tau}{2}$. Let $0=t_{0}<\cdots<t_{N}=1$ be a partition of the interval $[0,1]$, and define the operator $P_{N} \in \mathcal{L}(C([0,1]), P L([0,1]))$, where $P L([0,1]) \subseteq C([0,1])$ is the subspace of piecewise linear functions defined by setting $P_{N}[x]\left(t_{k}\right)=x\left(t_{k}\right)$ for each $k=0, \ldots, N$ with the partition sufficiently fine to ensure that $\left\|x-P_{N}(x)\right\| \leq \epsilon / 4$ for all $x \in K$. Let $L_{\delta}$ denote the closure of the set $P_{N}\left(K_{\delta}\right)$. Since $L_{\delta}$ lies in an $(N+1)$-dimensional subspace and is bounded and closed, it follows that $L_{\delta}$ is compact. It can be shown that $L_{\delta} \subseteq K_{\lambda}$, and hence $F_{\lambda, \mu}$ is well defined on $L_{\delta}$. By Theorem 4.1 for all $v \in L_{\delta}$ there exists an operator $S_{\lambda, \mu}: X \rightarrow C(T)$ in the form $S_{\lambda, \mu}=W Z Q G_{m}^{\star}$ such that $\left\|F_{\lambda, \mu}(v)-S_{\lambda, \mu}(v)\right\| \leq \frac{\tau}{2}$. We can now define the operator $S: X \rightarrow C(T)$ in the form $S=W Z Q G_{m}$, where $G_{m}=G_{m}^{\star} P_{N}$, by the equality $S(u)=S_{\lambda, \mu}\left(P_{N}[u]\right)$ for each $u \in K_{\delta}$.

\section{REFERENCES}

[1] B. Russell, On the notion of cause, Proc. Aristotelian Soc. 13 (1913), 1-25.

[2] R. E. A. C. Paley and N. Wiener, Fourier transforms in the complex domain, reprint of the 1934 original, Amer. Math. Soc. Colloq. Publ. 19, Providence, RI, 1987. MR 98a:01023

[3] Y. Foures and I. E. Segal, Causality and analyticity, Trans. Amer. Math. Soc. 78 (1955), 385-405. MR 16:1032d

[4] P. L. Falb and M. I. Freedman, A generalized transform theory for causal operators, SIAM J. Control 7 (1969), 452-471. MR 58:33192a

[5] J. C. Willems, Stability, instability, invertibility and causality, SIAM J. Control 7 no. 4 (1969), 645-671. MR 43:1689

[6] I. Z. Gohberg and M. G. Krein, Theory and Applications of Volterra Operators in Hilbert Space, Transl. of Math. Monographs, Vol. 24, Amer. Math. Soc., Providence, RI, 1970. MR 41:9041

[7] I. W. Sandberg and L. Xu, Uniform approximation of multidimensional myopic maps, IEEE Trans. on Circuits and Systems-1: Fund. Theory and Appl. 44 no. 6 (1997), 477-485. MR 97m:93024

[8] A. Torokhti and P. G. Howlett, On the constructive approximation of nonlinear operators in the modelling of dynamical systems, J. Austral. Math. Soc. Ser. B. 39 (1997), 1-27. MR 98c:41039

[9] P. G. Howlett and A. Torokhti, A methodology for the numerical representation of nonlinear operators defined on noncompact sets, Numer. Funct. Anal. and Optimiz. 18, no. 3-4 (1997), 343-365. MR 98i:47065

[10] P. G. Howlett and A. P. Torokhti, Weak interpolation and approximation of nonlinear operators on the space $\mathcal{C}([0,1])$, Numer. Funct. Anal. and Optimiz. 19, no. 9-10 (1998), 1025-1043. MR MR 99j:41003

[11] P. M. Prenter, A Weierstrass theorem for real, separable Hilbert spaces, J. Approx. Theory 3 (1970), 341-351. MR 55:6193

[12] V. J. Bruno, A Weierstrass approximation theorem for topological vector spaces, J. Approx. Theory 42 (1984), 1-3. MR 85j:41047

[13] G. Cybenko, Approximation by superpositions of a sigmoidal function, Math. Control Signals Systems 2 (1989), 303-314. MR 90m:41033. 
[14] I. K. Daugavet, On operator approximation by causal operators and their generalizations. II: Nonlinear case (Russian), Methods of Optimiz. and their Applic., Irkutsk. Sib. Energ. Institut (1988), 166-178.

[15] A. Torokhti and P. Howlett, On the Best Quadratic Approximation of Nonlinear Systems, IEEE Trans. on Circuits and Systems. Part I, Fundamental theory and applications, 48, No. 5 (2001), 595-602. MR 2003e:93015

[16] A. Torokhti and P. Howlett, Optimal Fixed Rank Transform of the Second Degree, IEEE Trans. on Circuits and Systems. Part II, Analog and Digital Signal Processing, 48, No. 3 (2001), 309-315.

Centre for Industrial and Applied Mathematics, University of South Australia, Mawson Lakes, SA 5095, Australia

E-mail address: p.howlett@unisa.edu.au

Centre for Industrial and Applied Mathematics, University of South Australia, Mawson Lakes, SA 5095, Australia.

E-mail address: a.torokhti@unisa.edu.au

Department of Applied Mathematics, University of Adelaide, Adelaide, SA 5005, Australia

E-mail address: cpearce@maths.adelaide.edu.au 